\title{
Editor's Announcement
}

Mater. Trans. 54 (2013) 1661-1666

The Barkhausen Effects and Nano-System Magnetizations in Fe

Shuji Obata

This paper has been retracted from Mater. Trans. by the author's request due to the following reason.

In the Fortran programming, Bohr magnetron constant of $\mu_{\mathrm{B}}=9.274 \times 10^{-24}$ was mistyped as $2.974 \times 10^{-24}$. This error caused the scale difference of 9.724/2.974 $(=1 / 0.32)$ times in the external field $H$. However, the error caused no influence on the other constants. Besides, this paper is expected to contain more quantitative arguments and the detailed comparison with the experimental data in Figs. 4, 6, 8 and 9. Therefore, the author requested to retract the above paper and to re-submit the revised paper with change of the title as "Computer Simulations on Barkhausen Effects and Magnetizations in Fe Nano-Structure Systems".

The author's apology for his honest error and the revision for improvement of the paper were accepted by the Editorial Committee.

The revised paper is published in Mater. Trans. 55 (2014) 1591-1598 after regular peer-reviewing by the Editorial Committee. 